\title{
Scaling up, Kinetic modeling, and Economic analysis of poly (3-hydroxybutyrate) production by Bacillus cereus isolate CCASU-P83
}

\author{
Noha S. Elsayed ${ }^{a^{*}}$, Khaled M. Aboshanab ${ }^{a}$, Mohammad M. Aboulwafa ${ }^{\text {a,b }}$, Mahmoud A. Yassien ${ }^{a}$, \\ Nadia A. Hassouna ${ }^{a}$ \\ a Department of Microbiology and Immunology, Faculty of Pharmacy, Ain Shams University, Cairo 11566, Egypt \\ ${ }^{\mathrm{b}}$ Faculty of Pharmacy, King Salman International University, Ras-Sedr, South Sinai, Egypt
}

\begin{abstract}
Polyhydroxyalkanoates (PHA) are environmentally friendly polymers produced by many bacteria under nutrientlimited conditions. However, their commercialization is hindered by production expenses. The present study aimed at cost-effective and efficient production of poly(3-hydroxybutyrate) (PHB) by Bacillus (B.) cereus isolate CCASUP83. Through one factor at time optimization study on shake flask, B. cereus CCASU-P83 produced about $50 \%$ polymer per dry weight after 48 hours incubation time. For better evaluation of the fermentation process, kinetic modeling using the Logistic and Leudking-piret models was applied. A preliminary economic analysis was carried out and leads to a $30.8 \%$ reduction in the total cost. In comparison to the findings obtained on the level of shake flask, scaling up to the bioreactor resulted in producing about 53\% PHB per dry weight after only 24 hours incubation. These models concluded that B. cereus produced PHB during the growth phase. Analysis of molecular weight of the produced polymer displayed a $26900 \mathrm{~g} / \mathrm{mole}$ molecular weight with a polydispersity index (PDI) of 1.1. In conclusion, B. cereus CCASU-P83 is a potential candidate for industrial production of PHB polymer using corn oil in a short incubation period which highly reduced the cost of the production process.
\end{abstract}

Keywords: Polyhydroxyalkanoates; Logistic; Leudking; Fermentation; optimization.

*Correspondence | Noha S. Elsayed; Department of Microbiology and Immunology, Faculty of Pharmacy, Ain Shams University, Cairo 11566, Egypt. Email: noha.yousef@pharm.asu.edu.eg

Citation | Elsayed NS, Aboshanab KM, Aboulwafa MM, Yassien MA, Hassouna NA, 2021. Scaling up, Kinetic modeling, and Economic analysis of poly (3-hydroxybutyrate) production by Bacillus cereus isolate CCASU-P83. Arch Pharm Sci ASU 5(1): 158-170

DOI: 10.21608/aps.2021.76831.1061

Print ISSN: 2356-8380. Online ISSN: 2356-8399.

Received 19 May 2021. Accepted 16 June 2021.

Copyright: ${ }^{\circledR} 2021$ Elsayed et al. This is an open-access article licensed under a Creative Commons Attribution 4.0 International License (CC BY 4.0), which permits unrestricted use, distribution, and reproduction in any medium, provided the original author(s) and source are credited.

Published by: Ain Shams University, Faculty of Pharmacy

\section{INTRODUCTION}

A lot of attention is directed towards increasing the productivity of poly (3hydroxybutyrate) (PHB), a plastic alternative, by different bacteria due to its high industrial potential. This polymer has similar properties to synthetic polymers besides biodegradability and biocompatibility which facilitate its utilization in biomedical implantation and controlled release preparations. However, the high production cost of PHB polymer is an obstacle in the road of commercial use of this polymer [1]. Accordingly, a lot of trials were carried out to decrease this high cost by selecting the suitable microorganism, optimization of small and large scale production, kinetic modeling, and characterization of the produced polymer [2]. Both Gram-positive and Gram-negative bacteria from various genera are identified to produce polyhydroxyalkanoates (PHA) polymers. Physiologically, these myriads of bacteria differ 
in the timing of PHA production. Some of them synthesize the polymer in the late log phase of the growth cycle and others in the stationary phase. However, all of them use it as a carbon source in starvation periods [3]. There are some bacterial species capable of producing the PHA polymers in an enormous amount suitable for industrial use for example Alcaligenes latus, Cupriavidus necator, and Pseudomonas putida [4, 5]. In addition to all of the previous examples, Bacillus species occupy a high rank in the industrial production of PHA biopolymer. They lack lipopolysaccharides which remove the burden of cumbersome product purification [6]. They have a high growth rate and excellent genetic stability [3]. The major drawback of using Bacillus species in PHA production is its sporulation which leads to PHA deposition [7]. Another drawback is the high cost of the raw materials used by Bacillus species which negatively impacts the applicability of large-scale production of PHA [8]. Thus, the development of an efficient submerged fermentation of Bacillus species using low-cost raw materials is crucial.

In our previous study, a lot of experiments were implemented to search for promising bacterial species capable of producing PHB polymer at an industrial scale. B. cereus isolate CCASU-P83 was selected owing to producing $13 \%$ PHB per dry weight after 48 hours of incubation using basal medium [9]. Therefore, this study aimed at investigating different environmental factors and culture conditions affecting PHB production by the respective isolate first on the shake flask followed by scaling up using a 14L bioreactor. Moreover, kinetic modeling and economic analysis were carried out to have new insights on the PHB production and its potential commercialization.

\section{MATERIALS AND METHODS}

\subsection{PHB production on shake flask}

B. cereus isolate CCASU-P83 (NCBI nucleotide accession code $\mathrm{KC} 876035)$ is a soil bacterium capable of producing PHB polymer [9]. This isolate was subcultured on LB agar, incubated at $37{ }^{\circ} \mathrm{C}$ and maintained as a $20 \%$ (vol/vol) glycerol stock preparation and deposited in Culture collection Ain Shams University (CCASU) which is listed in the World Data Centre for Microorganisms (WDCM) (http://www.wfcc.info/ccinfo/collection/by_id/11 86). For PHB production, the preparation of the pre-culture was carried out by culturing the bacteria into LB broth at $37{ }^{\circ} \mathrm{C}, 160 \mathrm{rpm}$, and 20 hours incubation. Then, the culture was carried out in the mineral salt medium (MSM) as shown in Table 1 where the inoculum size, shaking speed, temperature, and time was 5\% v/v, 200 rpm, $37^{\circ} \mathrm{C}$, and $48 \mathrm{~h}$, respectively.

\subsection{Different conditions affecting the PHB production}

Different aeration levels were tested by changing the ratio of culture medium to the Erlenmeyer flask headspace $(90,80$, and $60 \%$ aeration). Moreover, variable initial $\mathrm{pH}(4,5,7$, 8, and 9), inoculum sizes into MSM medium $(0.5,2,5$, and $10 \% \mathrm{v} / \mathrm{v})$, and incubation temperatures $\left(28,37\right.$, and $\left.40{ }^{\circ} \mathrm{C}\right)$ were investigated.

\subsection{Effect of various media ingredients}

\subsubsection{The carbon sources}

Various carbon sources were evaluated instead of glucose in the basal medium at the same concentration $(0.7 \% \mathrm{w} / \mathrm{v})$. The tested categories of carbon sources were monosaccharides, disaccharides sugars, sugar alcohol, polysaccharides, oils, and unrefined carbon sources such as Malt extract. Thereafter, the carbon source(s) gave maximum PHB production was tested at different concentrations $(0.4 \%, 0.7 \%, 1.5 \%, 2 \%$ and $4 \% \mathrm{w} / \mathrm{v})$ to select the optimum concentration. 


\subsubsection{The nitrogen sources}

Two different groups of nitrogen sources were tested organic and inorganic compounds. Different concentrations of the optimum nitrogen source were investigated.

\subsubsection{The multivalent minerals}

Five sets of MSM flasks were used: One flask is an MSM medium devoid of all minerals and the other four flasks contain individual minerals in the MSM medium.

\subsection{PHB production using the new medium}

The new medium (coded 83M, Table 1) was created based on the optimum results for every tested factor or condition as previously reported [10].

Table 1. Composition of MSM and newly formulated medium 83M used in PHB production

\begin{tabular}{|c|c|c|}
\hline Name of ingredient & Basal medium (MSM) & $\begin{array}{l}\text { Newly formulated medium } \\
(83 \mathrm{M})\end{array}$ \\
\hline $\begin{array}{l}\text { Carbon source } \\
\text { (amount/L) }\end{array}$ & Glucose (7 g) & Corn oil (7 mL) \\
\hline $\begin{array}{l}\text { Nitrogen source } \\
\text { (amount/L) }\end{array}$ & Ammonium chloride $(0.1 \mathrm{~g})$ & Ammonium chloride $(0.2 \mathrm{~g})$ \\
\hline Minerals (amount/L) & $\begin{array}{l}\mathrm{MgSO}_{4} \cdot 7 \mathrm{H}_{2} \mathrm{O}(0.2 \mathrm{~g}) \mathrm{CaCl}_{2} \quad(0.01 \mathrm{~g}) \text {, Ferrous } \\
\text { ammonium } \\
\text { sulphate }(0.06 \mathrm{~g}) \text {, trace elements solution* }(1 \mathrm{~mL})\end{array}$ & No Minerals \\
\hline $\begin{array}{l}\text { ommon ingredients } \\
\text { (amount/L) }\end{array}$ & $\mathrm{Na}_{2} \mathrm{HPO}_{4} \cdot 12 \mathrm{H}_{2} \mathrm{O}(10.2 \mathrm{~g}), \mathrm{KH}_{2} \mathrm{PO}_{4}(1.5 \mathrm{~g}), \mathrm{NaCl}$ & $(10 \mathrm{~g})$ \\
\hline
\end{tabular}

* -Trace elements solution contains (amount/L) $\left(\mathrm{CoCl}_{2} .6 \mathrm{H}_{2} \mathrm{O}(0.2 \mathrm{~g}), \mathrm{H}_{3} \mathrm{BO}_{3}(0.3 \mathrm{~g}), \mathrm{ZnSO}_{4} \cdot 7 \mathrm{H}_{2} \mathrm{O}(0.1 \mathrm{~g}), \mathrm{MnCl}_{2}\right.$ $.4 \mathrm{H}_{2} \mathrm{O}(30 \mathrm{mg}), \mathrm{NiCl}_{2}(10 \mathrm{mg}), \mathrm{CuSO}_{4} .5 \mathrm{H}_{2} \mathrm{O}(10 \mathrm{mg})$.

- MSM and 83M media were sterilized by autoclaving. Glucose and trace elements solution were filter sterilized and were aseptically added to the autoclaved media with the indicated concentration.

\subsection{Economic analysis}

To preliminary evaluate the change in cost after applying the new medium on shake flask, an economic analysis [11] was carried out by calculating these parameters:

Change in total cost $=$ Total cost of a new medium (P2 )-Total cost in basal medium (P1 ) [11].

\subsection{Scaling up}

PHB production was carried out in a $14 \mathrm{~L}$ Bioflo 310 glass bioreactor (New Brunswick
Scientific, Edison, NJ, USA. The special conditions for the bioreactor were $1 \mathrm{vvm}$ aeration (4 SLPM), $100 \%$ oxygen saturation, and uncontrolled $\mathrm{pH}$. Different samples were collected for measuring the PHB concentration, biomass, PHB percentage per dry weight, and corn oil utilization [12].To study the effect of aeration, other fermentation runs were carried out using the same conditions listed above except at different aeration rates of $0.5,2$, and $4 \mathrm{vvm}$. 


\subsection{Kinetic modeling}

The fermentation process was described using two kinetics models; one describing cell growth and the other describing product formation. This was applied to the best fermentation run.

\subsubsection{Cell growth kinetic model}

A substrate-independent model described by logistic equation [13] was formulated as follows:

$$
X=\frac{x^{2} \exp \left(\mu_{m} t\right)}{1-\left(\frac{x^{\circ}}{x_{m}}\right)\left(1-\exp \left(\mu_{m} t\right)\right.}
$$

where $\mu$ is the specific growth rate, $\mu \mathrm{m}$ is the maximum specific growth rate ( $\mathrm{h}-1)$, $\mathrm{x}$ is biomass concentration concerning time $(\mathrm{g} / \mathrm{L})$ and $\mathrm{XM}$ is maximum cell dry weight concentration $(\mathrm{g} / \mathrm{L})$.

\subsubsection{Product formation kinetic model}

The biosynthesis of PHB can be represented by Luedeking-Piret type model [14]. The product formation rate is described as:

$P_{t}=P_{s}+\alpha\left[\frac{X_{\bullet} \varepsilon^{\mu_{m} t}}{\left(1-\left(\frac{X_{a}}{X_{m}}\right)\left(1-\varepsilon^{\mu_{m} t}\right)\right.}-X s\right]+\beta\left(\frac{X_{m}}{\mu_{m}}\right) \ln \left[1-X_{0} / X_{m}\left(1-e^{\mu_{m} t}\right)\right]$

[14]

Where $\alpha$ is growth-associated constant, $\beta$ is non-growth-associated constant, $\mathrm{x}$ is biomass concentration and $\mathrm{dx} / \mathrm{dt}$ is the rate of growth.

Kinetic model parameters were estimated by applying experimental data to the proposed models using software Graph Pad Prism version 5 by using nonlinear least-squares curve fitting with $95 \%$ confidence interval.

\subsection{Molecular weight measurement}

Molecular weight data were obtained by gel permeation chromatography (GPC) with a refractive index detector in the National research center, Dokki, Egypt. The extracted polymer was dissolved in dimethylformamide, filtered using a $0.45 \mu \mathrm{m}$ syringe filter, and sent for measurement. A report is provided for each sample stating three important values: the number average molecular weight $(\mathrm{M} \mathrm{N})$, the weight average molecular weight $(\mathrm{M} \mathrm{w})$, and the polydispersity index (PDI).

\subsection{Analytical Methods}

\subsubsection{Biomass determination}

Biomass was expressed as dry cell weight which was derived from a calibration curve created between optical density (OD640 nm) and dry cell weight of the isolate CCASU-P83 [10].

\subsubsection{PHB concentration determination}

It was determined using the spectrophotometric method [15]. The extracted PHB polymer was converted to crotonic acid by concentrated sulphuric acid and was measured at $235 \mathrm{~nm}$.

\section{RESULTS AND DISCUSSION}

\subsection{Shake flask production}

\subsubsection{Aeration and incubation temperature effect on PHB production}
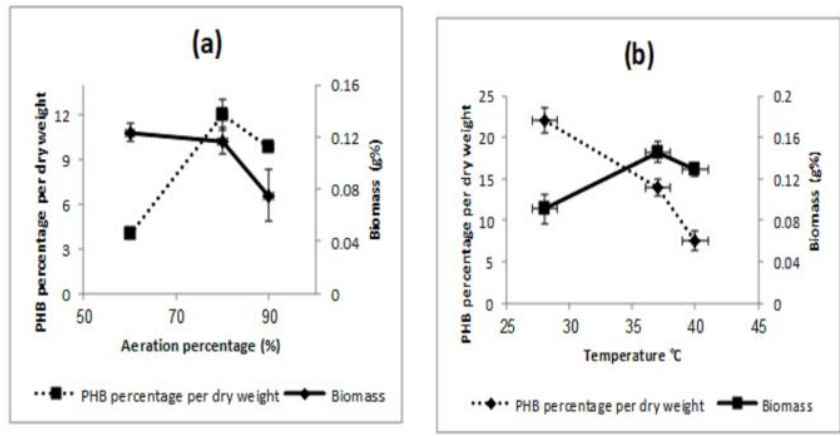

Fig 1. Effect of aeration (a) and incubation temperature (b) on PHB percentage per dry weight and biomass in B. cereus isolate $\mathrm{P} 83$

B. cereus isolate CCASU-P83 achieved its highest PHB percentage per dry weight using $80 \%$ aeration and at $28{ }^{\circ} \mathrm{C}$ (Fig. 1). For the aeration level, these results coincide with many studies supporting that oxygen is a very important trigger for PHB production [16, 17]. Since the optimum incubation temperatures range 
for PHB production is from $25-35{ }^{\circ} \mathrm{C}[18,19]$, thus our results fall in the correct zone.

\subsubsection{Effect of $\mathrm{pH}$ and inoculum size}

Results of testing different $\mathrm{pH}$ and inoculum sizes revealed that the highest PHB percentage per dry weight was established using $\mathrm{pH} 4$ and $0.5 \%$ inoculum size. However, they were not selected as optimum conditions in further studies because this upheaval is due to a reduction in biomass rather than an increase in PHB accumulation. Thus, $\mathrm{pH} 7$ and 5\% inoculum size were used as optimum conditions because the organism accumulated the highest PHB polymer under these conditions.

\subsubsection{Effect of different carbon and nitrogen sources on PHB production}

Effect of different carbon sources on PHB productivity of the test isolate (Table 2) showed that the highest PHB percentage per dry weight was achieved using corn oil and paraffin oil. A lot of studies advocated the use of oil as an optimum carbon source [20, 21]. Borah and his coworkers (2002) mentioned that carbon sources either target biomass formation or PHB production [16]. This was brightly observed in the present study where glycerol, sucrose, maltose, and fructose enhanced the biomass formation only. Since paraffin oil and corn oil gave the best results so they were tested at different concentrations. It was observed that the best concentration for both oils was $1.5 \% \mathrm{v} / \mathrm{v}$ based on the high PHB percentage per dry weight previously determined [10]. Consequently, corn oil $(0.7 \%)$ was chosen as the optimum carbon source.

Table 2. Effect of replacement of glucose with other carbon sources on PHB percentage per dry weight and biomass of $B$. cereus isolate CCASU-P83

\begin{tabular}{lcc}
\hline $\begin{array}{l}\text { Carbon source } \\
(\mathbf{0 . 7} \%)\end{array}$ & $\begin{array}{c}\text { PHB percentage per dry } \\
\text { weight }\end{array}$ & Biomass (g\%) \\
\hline Glucose (control) & 13 & 0.1 \\
Galactose & 10 & 0.0936 \\
Fructose & 6 & 0.126 \\
Maltose & 2 & 0.1465 \\
Lactose & 20 & 0.0233 \\
Sucrose & 2.5 & 0.1728 \\
Mannitol & 16 & 0.0611 \\
Glycerol & 4.4 & 0.1928 \\
Starch & 14 & 0.0848 \\
Paraffin oil & 27 & 0.0414 \\
Corn oil & 24 & 0.051 \\
\hline
\end{tabular}

In the present study, the limitation of nitrogen was chosen to trigger PHB production because ammonia is considered a pivotal factor for the uncoupling of growth and PHB [22]. The results of the effect of different nitrogen sources on PHB percentage per dry weight and biomass formation are displayed in Table 3. It was found that both ammonium chloride and peptone produced a 
comparable high PHB percentage per dry weight. Trakunjae et al (2021) tested both inorganic and organic nitrogen sources for optimum production of PHB by Rhodococcus and they reported that the inorganic nitrogen source, potassium nitrate, gave the best results [23]. On the other hand, Tripathi et al (2012) advocated the use of urea for optimum production of PHB by Pseudomonas aeruginous [15]. After testing different concentrations of both ammonium chloride and peptone, about $0.1 \mathrm{~g} / \mathrm{L}$ of ammonium chloride attained the highest PHB percentage per dry weight.

Table 3. Effect of replacement of ammonium chloride with different nitrogen sources on PHB productivity per dry weight and biomass of B. cereus isolateCCASU-P83

\begin{tabular}{lcc}
\hline Nitrogen source $(\mathbf{0 . 1} \mathbf{g} / \mathbf{L})$ & $\begin{array}{c}\text { PHB percentage per dry } \\
\text { weight }\end{array}$ & Biomass $(\mathbf{g} \%)$ \\
\hline Ammonium chloride & 13 & 0.1165 \\
(control) & 11 & \\
Yeast extract & 12.8 & 0.0504 \\
Peptone & 2.2 & 0.0983 \\
Beef extract & 3.6 & 0.1218 \\
Urea & 9 & 0.1195 \\
Tryptone & 2 & 0.0442 \\
Potassium nitrate & 5 & 0.0482 \\
Ammonium nitrate & & 0.2141 \\
\hline
\end{tabular}

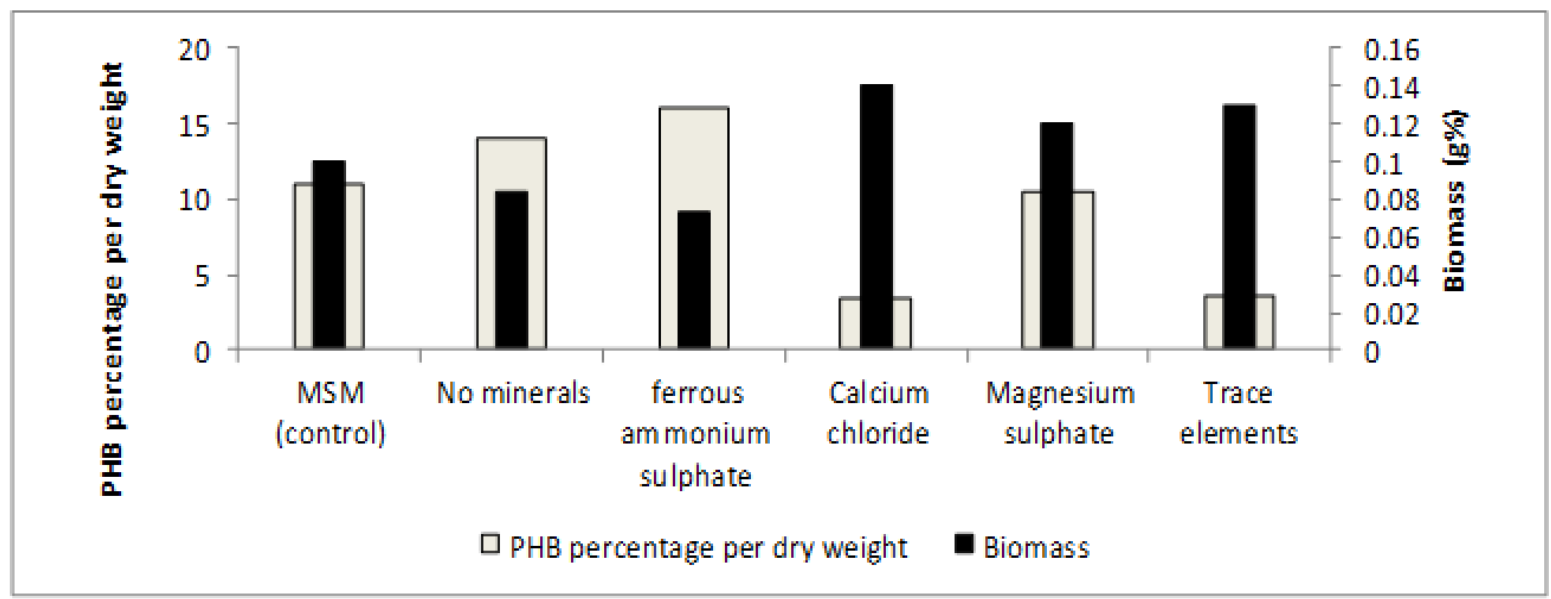

Fig 2. Effect of minerals on PHB percentage per dry weight and biomass formation of B. cereus isolate P83

\subsubsection{Effect of minerals on PHB production}

As shown in Fig. 2, the maximum PHB percentage per dry weight was achieved using
MSM devoid of all minerals and that containing ferrous ammonium sulfate only. However ferrous ammonium sulfate decreased biomass in 
comparison to MSM devoid of minerals. Thus, MSM medium devoid of minerals were used for further studies. This agreed with what Sangkharak and Poonsuk (2008) noticed that both calcium chloride and magnesium sulfate have a negligible effect on PHB production [22].

\subsubsection{Time course of PHB production by $B$. cereus using newly formulated medium $(83 \mathrm{M})$}

After gathering all the optimum factors in the new medium $(83 \mathrm{M})$, the results were fascinating. B. cereus isolate CCASU-P83 showed a pronounced increase in PHB percentage per dry weight from $13 \%$ to $50 \%$ (Table 4). This implies that the maximum $\mathrm{PHB}$ production percentage per dry weight was about 4 fold higher in the new medium (83 M) compared to basal medium after equal incubation periods of $48 \mathrm{~h}$. This was higher with what reported by Valaprill et al (2007) $38 \%$ PHA production for B.cereus using glucose [24]. On the other side, Sangkharak and Prasertsan (2012) observed 64\% PHA production using $B$. cereus using Palm oil effluent after $96 \mathrm{~h}$ of incubation [25]. However, our results are better in terms of a short incubation period (48 $\mathrm{h}$ ) rather than $(96 \mathrm{~h})$ which is more cost-effective. Furthermore, Chaudhry et al (2011) used corn oil as a feeding substrate for different Pseudomonas species for PHA production and about 35\% per dry weight was the best achievement [26].

Table 4. The maximum productivity and PHB production percentage per dry weight of $\mathbf{B}$. cereus isolate in basal and newly formulated media

\begin{tabular}{lcc}
\hline Isolate & Medium applied & $\begin{array}{c}\text { Maximum PHB production } \\
\text { percentage per dry weight (in } \\
\text { hours) }\end{array}$ \\
\hline B. cereus CCASU-P83 & Basal medium (MSM) & $13 \%(48 \mathrm{~h})$ \\
& $83 \mathrm{M}$ & $50 \%(48 \mathrm{~h})$ \\
\hline
\end{tabular}

\subsection{Economic analysis}

By applying a simple economic estimation of the total production cost of both the basal medium and the new media, about a $30.8 \%$ reduction in total cost was calculated as shown in Table 5 after using the new medium. This is an incredible reduction in the cost of PHB production by this isolate which predisposes to a cost-effective process. The reason behind this reduction is the usage of cheap carbon sources such as corn oil instead of glucose. The cost of carbon source contributes to $28 \%$ of the total cost of PHA production so removing this obstacle paves the way for proper commercialization [27]. Naranjo and his coworkers (2013) stated also a reduction in the price of $\mathrm{PHB}$ production upon replacing glucose with glycerol [6].

\subsection{Scaling up}

The use of the shake flask conditions $\left(28{ }^{\circ} \mathrm{C}\right.$ temps, $200 \mathrm{rpm}$ agitation, $1 \mathrm{vvm}$ aeration) in $14 \mathrm{~L}$ laboratory bioreactor led to a 1.4-fold increase in PHB production in a shorter incubation time than that of shake flask (24 rather than $48 \mathrm{~h}$ ) as shown in Fig. 3. Batch fermentation is known to enhance the production of PHB polymer [27]. Further studies were carried out for the respective isolate to study the effect of aeration on PHB production. It was found that upon decreasing aeration to $0.5 \mathrm{vvm}$, the PHB production and biomass decreased significantly. Peña and his coworkers (2014) pointed that decreasing 
aeration decreases the volumetric PHB production which agrees with our results [28]. On the other hand, increasing the aeration level to 2 vvm (Fig. 4) led to an increase in PHB production with a slight decrease in biomass than that obtained using $1 \mathrm{vvm}$ aeration. Accordingly, increasing the aeration level from $1 \mathrm{vvm}$ to 2 vvm was associated with an increase in the PHB percentage per dry weight from $49 \%$ to $53 \%$. However, a further increase in aeration level to 4 vvm was associated with a decrease in PHB production and biomass. From the obtained results, the optimum aeration level for PHB production by $B$. cereus isolate CCASU-P83 was $2 \mathrm{vvm}$ which was used for further studies. In a nutshell, B. cereus isolate CCASU-P83 produced $0.266 \mathrm{~g} / \mathrm{L}$ with a polymer content of $53 \%$ per dry weight which was higher than that produced by different Bacillus species in other studies [29-31].

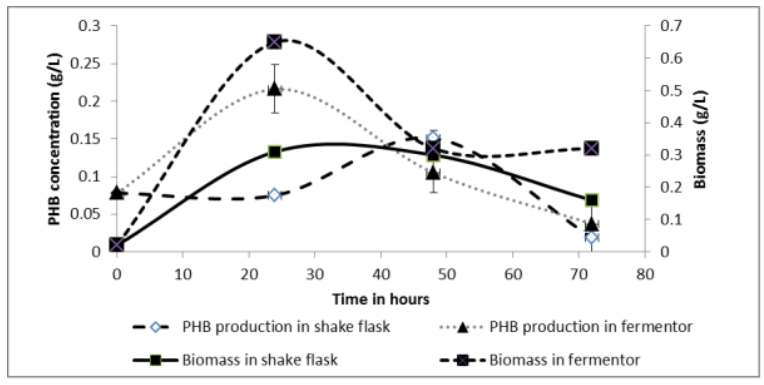

Fig 3. PHB production and biomass formation profiles of $B$. cereus isolate P83 in the shake flask and in the bioreactor using the same production media and culture conditions

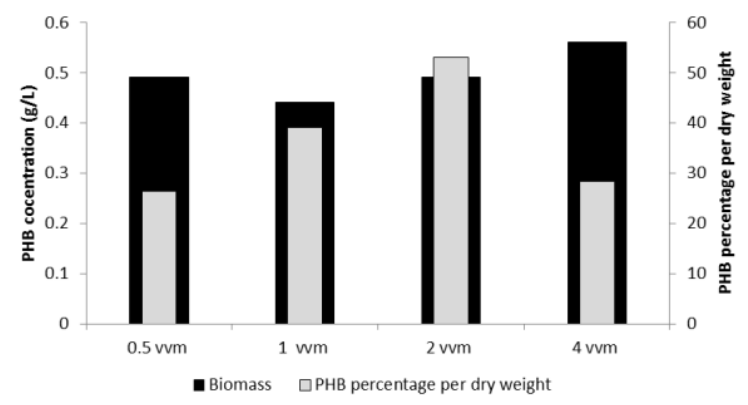

Fig 4. Maximum PHB percentage per dry weight by $B$. cereus isolate P83 in the laboratory bioreactor at different aeration rates after $24 \mathrm{~h}$ of fermentation. Conditions applied: uncontrolled initial $\mathrm{pH}$ of 7.2 ; temperature of $28{ }^{\circ} \mathrm{C}$; agitation rate of $200 \mathrm{rpm}$; and inoculum size $5 \% \mathrm{v} / \mathrm{v}$

\subsection{Kinetic modeling}

The ability of Bacillus cereus isolate to produce $\mathrm{PHB}$ polymer in $24 \mathrm{~h}$ intrigued us to search whether the production of PHB was during the growth phase or stationary phase. Thus we used mathematical modeling which is mainly used to describe the microbial performance during the fermentation process by mathematical equations [32]. Two mathematical models, Logistic and Leudking piret, were used to describe microbial growth and product formation kinetics.

Logistic model mostly used to describe the bacteria growth kinetics during polymer production [14]. This model implies a directly proportional relationship between the rate of cellular growth and cell mass concentration. According to the obtained results of kinetic modeling, the used logistic model (Equation 1) fitted well to experimental culture conditions producing a good predictive model for microbial growth of the tested isolate with an $\mathrm{r}^{2}$ value of approximately 0.99 (Fig. 5A). The values of maximum specific growth rate and maximum biomass are shown in Table 5.
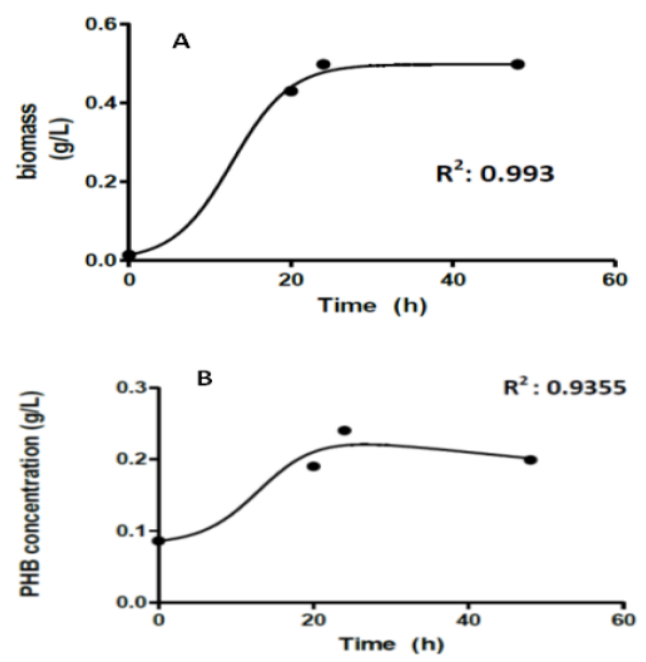

Fig 5. A) Cell growth kinetic model curves of fermentation for $B$. cereus isolate P83 in comparison to experimental data. B) Product synthesis kinetic model curves of fermentation for $B$. cereus isolate P83 in comparison to 
experimental data. The dots represent the experimental results and the lines represent the data determined by the described kinetic model

The Luedeking-Piret equation was applied in the present study to describe the microbial behavior for polymer production. The Luedeking-Piret model categorizes the relationship between the product and the microorganism growth into Class 1 where microorganism growth is directly proportional to product formation where $\alpha>0, \beta=0$; Class 2 where the partial relationship is found between the two systems so $\alpha>0, \beta>0$; and Class 3 where no relationship between the product and the microorganism growth so $\alpha=0, \beta>0$ [33].

In the present study, the obtained results revealed that the Luedeking-Piret model fitted very well to the experimental data (Fig. 5B), and the growth associated constant $(\alpha)$ was higher than the non-growth associated constant $(\beta)$ in this isolate, so the polymer is growth associated product; class 1. Growth-associated production means that the rate of polymer production parallels the bacterial growth rate. Many studies confirmed the growth associated nature of PHB production where Khan et al. (2013) reported this phenomenon in Cupriavidus necator H16 [34]. Moreover, Yang et al (2006) mentioned that Zoogloea sp. GY3 produces PHA during growth [25].

\subsection{Molecular weight measurement}

Molecular weight is a fundamental property of PHA polymer. There is a direct relationship between the polymer's mechanical strength and its molecular weight [35]. The values of the molecular weight measured are listed in Table 6. Cuellar et al. (2011) reported that PHB average molecular weight ranges from 104 to 106 $\mathrm{g} / \mathrm{mole}$ [36]. Another important property of a polymer is its polydispersity index $(\mathrm{Mw} / \mathrm{Mn})$. In this study, low polydispersity was recovered by B. cereus isolate CCASU-P83 (1.1) which supports the usage of PHB polymer recovered in biomedical applications.

Table 5. Parameter values calculated using Logistic and Luedeking-Piret equation

\begin{tabular}{|c|c|}
\hline Parameter & Value \\
\hline \multicolumn{2}{|c|}{ Logistic model (Equation 1) } \\
\hline $\mathrm{X}^{\circ}(\mathrm{g} / \mathrm{L}) \mathrm{a}$ & 0.014 \\
\hline $\mathrm{Xm}(\mathrm{g} / \mathrm{L}) \mathrm{b}$ & 0.498 \\
\hline$\mu \mathrm{m}(\mathrm{h}-1) \mathrm{c}$ & 0.2781 \\
\hline \multicolumn{2}{|c|}{ Luedeking-Piret model (Equation2) } \\
\hline$\alpha$ (Growth associated constant) & 0.3168 \\
\hline$\beta$ (Non-growth associated constant) & 0.002197 \\
\hline
\end{tabular}

a $\mathrm{X}^{\circ}$ : initial biomass, $\mathrm{b} X \mathrm{~m}$ : Maximum biomass, $\mathrm{c} \mu \mathrm{m}$ : Maximum specific growth rate 
Table 6. The molecular weight of PHB synthesized by B. cereus isolate CCASU-P83

\begin{tabular}{|c|c|c|c|c|}
\hline Isolate & MW & Mn & PDI & NP \\
\hline B. cereus isolate CCASU-P83 & $26900 \mathrm{~g} / \mathrm{mole}$ & $24000 \mathrm{~g} / \mathrm{mole}$ & 1.1 & $3.3 * 1018$ \\
\hline
\end{tabular}

MW, weight average molecular weight; Mn, number average molecular weight; PDI, polydisperisity index; Np, no. of polymer chains per liter

\section{Conclusion}

The soil bacterium $B$. cereus CCASU-P83 produces $\mathrm{PHB}$ polymer using corn oil and ammonium chloride as carbon and nitrogen sources, respectively. Under optimum conditions obtained through experimentation, this strain can accumulate $50 \%$ PHB per dry cell weight. Using the optimized medium there was an improvement both technically and economically. Technically the optimized medium improved the production from $13 \%$ to $50 \%$ per dry cell weight and economically there was a $30.8 \%$ reduction in total cost. Furthermore, a higher PHB percentage was attained using a $14 \mathrm{~L}$ bioreactor in a shorter incubation period. Finally, the mathematical modeling of both growth and polymer production concluded the growth associated production of PHB polymer using B. cereus isolate CCASUP83.

\section{Declarations}

\section{Ethics approval and consent to participate}

Not applicable

\section{Data availability}

Data supporting the conclusion of the study is available in the manuscript and the nucleotide DNA sequencing of the $16 \mathrm{~S}$ ribosomal RNA is available under the NCBI GenBank accession code, KC876035.

\section{Conflict of interest}

The authors declare that they have no competing interests

\section{Funding Statement}

This research did not receive any specific grant from funding agencies in the public, commercial, or not-for-profit sectors.

\section{Author contributions}

All authors contributed extensively to the work presented in this paper. NS designed, performed the experiments, and wrote the manuscript. KM, MA, and MM designed the experiments, analyzed data, and revised the manuscript. NH supervised and revised the manuscript. All authors discussed the results and commented on the manuscript at all stages

\section{Acknowledgments}

The authors acknowledge Dr. Manal Metwally, Professor of Economics in the Faculty of economics and political sciences in Cairo for her appreciated help in the economic analysis part. The authors are very thankful to Dr. Ghadir EL-Housseiny, Ph.D., for her valued assist in the kinetic modeling part.

\section{Consent to publish}

Not applicable

\section{Availability of data and materials}

All data generated or analyzed during this study are included in this published article in the main manuscript.

\section{Competing interests}


No competing interests were declared by the authors.

\section{REFERENCES}

1. Chen G-Q, Chen X-Y, Wu F-Q, Chen J-C. Polyhydroxyalkanoates (PHA) toward cost competitiveness and functionality. Adv Indust and Eng Poly Res. 2020; 3: 1-7. https://doi.org/https://doi.org/10.1016/j.aiepr.2 019.11 .001

2. Chen G-Q, Jiang X. Engineering bacteria for enhanced polyhydroxyalkanoates (PHA) biosynthesis, Synth Syst Biotechnol. 2017; 2: 192-7. https://doi.org/https://www. sciencedirect.com/science/article/pii/S240580 5X17300819

3. Mohapatra S, Maity S, Dash H, Das H, Pattnaik S, Rath C, et al. Bacillus, and biopolymer: Prospects and challenges. Biochem Biophys Rep. 2017; 12: 206-13. https://doi.org/10.1016/j.bbrep.2017.10.001

4. Gerngross, T., Snell, KD, Peoples O., Sinskey A. Overexpression and Purification of the Soluble Polyhydroxyalkanoate Synthase from Alcaligenes eutrophus: Evidence for a Required Posttranslational Modification for Catalytic Activity. Biochemistry. 1994; 33: 931

1-9320. https://doi.org/https://doi.org/10.1021/bi00197 a035

5. Beckers V, Poblete-Castro I, Tomasch J, Wittmann C. Integrated analysis of gene expression and metabolic fluxes in PHAproducing Pseudomonas putida grown on glycerol. Microb Cell Fact. 2016; 15(73). https://doi.org/10.1186/s12934-016-0470-2

6. Naranjo J, Posada JA, Higuita JC, Cardona CA. Valorization of glycerol through the production of biopolymers: The PHB case using Bacillus megaterium. Bioresource Technology. 2013; 133: 38-44. https://doi.org/10.1016/j.biortech.2013.01.129

7. Chen G-Q. Plastics Completely Synthesized by Bacteria: Polyhydroxyalkanoates. In: Plastics from bacteria. Springer; 2009. p. 1737.

8. Choi J, Lee SY. Factors affecting the economics of polyhydroxyalkanoate production by bacterial fermentation. Appl Microbiol Biotechnol., 1999; 51: 13-21. https://doi.org/10.1007/s002530051357

9. Elsayed N, Aboulwafa M, Aboshanab Khaled, Hassouna N. PHB production in Azomonas, Acinetobacter and Bacillus species: Isolation, screening, and identification. Archives of clinical microbiology. 2013; 4(5). https://doi.org/10.3823/272.

10. Noha S. Elsayed, Khaled M. Aboshanab, Mohammad M. Aboulwafa, Nadia A. Hassouna. Cost-effective production of the bioplastic poly- $\beta$-hydroxybutyrate using Acinetobacter baumannii isolate P39. JMBFS 2016; 5(6): 553-556. doi: 10.15414/jmbfs.2016.5.6.552-556

11. Baumol WJ, Blinder AS. Economic principles and policy. Market and the price system. 2006. $195-259$ p.

12. Leitermann, F, Syldatk C, Hausmann R. Fast quantitative determination of microbial rhamnolipids from cultivation broths by ATRFTIR Spectroscopy. J Biol Eng. 2008; 2(13). https://doi.org/10.1186/1754-1611-2-13

13. Khanafari A, Akhavan Sepahei A, Mogharab M. Production and recovery of Poly-BHydroxybutirate from whey Degradation by Azotobacter. Iranian Journal of Environmental Health Science \& Engineering. 2006; 3(3). https://doi.org/10.1016/j.bej.2013.10.020

14. Dhanasekar R, Viruthagiri T, Sabarathinam P. Poly(3-hydroxy butyrate) synthesis from a mutant strain Azotobacter vinelandii utilizing 
glucose in a batch reactor. Biochem Eng J. 2003; 16: 1-8. https://doi.org/10.1016/S1369703X(02)00176-6

15. Tripathi AD, Joshi TR, Srivastava SK, Darani KK, Khade S, Srivastava J. Effect of nutritional supplements on bio-plastics (PHB) production utilizing sugar refinery waste with potential application in food packaging. Preparative Biochemistry and Biotechnology. 2019;

https://doi.org/10.1080/10826068.2019.15919 82

16. Borah B, Thakur P, Nigam J. The influence of nutritional and environmental conditions on the accumulation of poly-b-hydroxybutyrate in Bacillus mycoides RLJ B-017. J Appl Microbiol. 2002; 92(4): 776-83. https://doi.org/10.1046/j.1365-

2672.2002.01590.x

17. Pandian S, Deepak V, Kalishwarala K, Rameshkumar N, Jeyaraj M, Gurunathan S. Optimization and fed-batch production of PHB utilizing dairy waste and seawater as nutrient sources by Bacillus megaterium SRKP-3. Bioresour Technol. 2010; 101(2): 705-11. https://doi.org/10.1016/j.biortech.2009.08.040

18. Bhuwal AK, Singh G, Aggarwal NK, Goyal V, Yadav A. Poly- $\beta$-hydroxybutyrate production and management of cardboard industry effluent by new Bacillus sp. NA10. Bioresources and Bioprocessing. 2014; 1(9). https://doi.org/10.1186/s40643-014-0009-5

19. Elshikh M, Funston S, Chebbi A, Ahmed S, Marchant R, Banat IM. Rhamnolipids from non-pathogenic Burkholderia thailandensis E264: Physicochemical characterization, antimicrobial and antibiofilm efficacy against oral hygiene related pathogens. New Biotechnol. 2017 May 25; 36: 26-36. https://doi.org/10.1016/j.nbt.2016.12.009
20. Fukui T, Doi Y. Efficient production of polyhydroxyalkanoates from plant oils by Alcaligenes eutrophus and its recombinant strain. Appl Microbiol Biotechnol. 1998; 49(3): $333-6$. https://doi.org/10.1007/s002530051178.

21. Verlinden R, Hill D, Kenward M, Williams C, Piotrowska-Seget Z, Radecki I. Production of polyhydroxyalkanoates from waste frying oil by Cupriavidus necator. AMB Express. 2011; 1(1): 11. https://doi.org/10.1186/21910855-1-1

22. Sangkharak K, Poonsuk P. Nutrient optimization for production of polyhydroxybutyrate from halotolerant photosynthetic bacteria cultivated under aerobic-dark condition. Electron $\mathrm{J}$ of Biotechnol. 2008; 2 11(3). https://doi.org/10.2225/vol11-issue3-fulltext2.

23. Trakunjae C, Boondaeng A, Apiwatanapiwat W, Kosugi A, Arai T, Sudesh K, et al. Enhanced polyhydroxy butyrate (PHB) production by newly isolated rare actinomycetes Rhodococcus sp. strain BSRT1-1 using response surface methodology. Sci Rep. 2021 Jan 21; 11(1): 1896. https://doi.org/10.1038/s41598-02181386-2

24. Valappil S, Misra S, Boccaccini A, Keshavarz T, Bucke C, Roy I. Large-scale production and efficient recovery of PHB with desirable material properties, from the newly characterized Bacillus cereus SPV. Journal of Biotechnology. 2007; 132: 251-8. https://doi.org/10.1016/j.jbiotec.2007.03.013.

25. Sangkharak K, Prasertsan P. Screening and identification of polyhydroxyalkanoates producing bacteria and biochemical characterization of their possible application. $\mathbf{J}$ Gen Appl Microbiol. 2012; 58(3): 173-82. https://doi.org/10.2323/jgam.58.173 
26. Chaudhry WN, Jamil N, Ali I, Ayaz MH, Hasnain S. Screening for polyhydroxyalkanoate (PHA)-producing bacterial strains and comparison of PHA production from various inexpensive carbon sources. Ann Microbiol. 2011 Sep 1; 61(3): 623-9. https://doi.org/10.1007/s13213-0100181-6

27. Gamal RF, Abdelhady HM, Khodair TA, ElTayeb TS, Hassan EA, Aboutaleb KA. Semiscale production of PHAs from waste frying oil by Pseudomonas fluorescens S48. Braz J Microbiol Publ Braz Soc Microbiol. 2013 Oct $30 ; \quad 44(2)$ : 539-49. https://doi.org/10.1590/S151783822013000200034

28. Peña C, López S, García A, Espín G, RomoUribe A, Segura D. Biosynthesis of poly- $\beta$ hydroxybutyrate (PHB) with a high molecular mass by a mutant strain of Azotobacter vinelandii (OPN). Ann Microbiol. 2014; 64: 39-47. https://doi.org/10.1007/s13213-0130630-0

29. Yilmaz M, Soran H, Beyatli Y. Determination of poly- $\beta$-hydroxybutyrate (PHB) production by some Bacillus spp. World J Microbiol Biotechnol. 2005 Jun 1; 21(4): 565-6. https://doi.org/10.1007/s11274-004-3274-1

30. Patel SKS, Singh M, Kalia VC. Hydrogen and Polyhydroxybutyrate Producing Abilities of Bacillus spp. From Glucose in Two-Stage System. Indian J Microbiol. 2011 Oct 7; 51(4): 418. https://doi.org/10.1007/s12088011-0236-9

31. Olubusola O, Adeola O. Production and characterization of polyhydroxyalkanoate acid from Bacillus thuringiensis using different carbon substrates. Int J of Biolog Macromol. 2017; 104: 407-13. doi: 10.1016/j.ijbiomac.2017.06.041.

32. Panda I, Balabantaray S, Sahoo SK, Patra N.
Mathematical model of growth and polyhydroxybutyrate production using microbial fermentation of Bacillus subtilis. Chem Eng Commun. 2018 Feb 1; 205(2): 249-56.

https://doi.org/10.1080/00986445.2017.13849 23

33. Yang J, Huang J, Ni J. Mathematical modeling of the batch fermentation of Zoogloea sp. GY3 is used for synthesizing polyhydroxyalkanoates. J of Chem Technol and Biotechnol. 2006; 81: 789-93. https://doi.org/https://doi.org/10.1002/jctb.144 7

34. Khan M, Prasad D., Abdullah H, Batcha A. Kinetic Analysis on Cell Growth and Biosynthesis of Poly (3-Hydroxybutyrate) (PHB) in Cupriavidus Necator H16. International Journal of Bioscience, Biochemistry, and Bioinformatics, 2013; 3(5): 516-9. https://doi.org/10.7763/IJBBB.2013.V3.268

35. Tsuge T. Fundamental factors determining the molecular weight of polyhydroxyalkanoate during biosynthesis. Poly J. 2016; 48:1051-7. https://doi.org/10.1038/pj.2016.78

36. Cuellar MRL, Flores JA, Rodríguez G, Guevaraa P. Production of polyhydroxyalkanoates (PHAs) with canola oil as carbon source. International Journal of Biosciences. 2011; 48: 74-80. https://doi.org/10.1016/j.ijbiomac.2010.09.016 